\title{
Catechol-Containing Compounds are a Broad Class of Protein Aggregation Inhibitors: Redox State is a Key Determinant of the Inhibitory Activities
}

\section{Paul Velander}

Virginia Tech: Virginia Polytechnic Institute and State University

Ling Wu

Virginia Tech: Virginia Polytechnic Institute and State University

Sherry B. Hildreth

Virginia Tech: Virginia Polytechnic Institute and State University

Nancy J. Vogelaar

Virginia Tech: Virginia Polytechnic Institute and State University

Biswarup Mukhopadhyay

Virginia Tech: Virginia Polytechnic Institute and State University

Shijun Zhang

Virginia Commonwealth University

Richard F. Helm

Virginia Tech: Virginia Polytechnic Institute and State University

Bin Xu ( $\square$ binxu@vt.edu )

Virginia Tech: Virginia Polytechnic Institute and State University https://orcid.org/0000-0002-04909540

\section{Research article}

Keywords: Amyloid Inhibitors, Library Screening, Catechol-Containing Compounds, Redox Regulation, Structure-Activity Relationship

Posted Date: December 14th, 2020

DOl: https://doi.org/10.21203/rs.3.rs-122859/v1

License: (c) (1) This work is licensed under a Creative Commons Attribution 4.0 International License. Read Full License 


\section{Abstract}

Background: A range of neurodegenerative and related aging diseases, such as Alzheimer's disease, Parkinson's disease, and type 2 diabetes, are linked to toxic protein aggregation. Yet the mechanisms of protein aggregation inhibition by small molecule inhibitors remain poorly understood, in part because most protein targets of aggregation assembly are partially unfolded or intrinsically disordered, which hinders detailed structural characterization of protein-inhibitor complexes and structural-based mechanistic elucidation.

Methods: Herein we employed a small molecule screening approach to identify inhibitors against three prototype amyloidogenic proteins in neurodegeneration and related proteinopathies: amylin, $A b$ and tau. We further systematically investigated selected class of inhibitors under aerobic and anaerobic conditions to uncover a key determinant of the inhibitory activities.

Results: One remarkable class of inhibitors identified from all three parallel screenings against different amyloidogenic proteins was catechol-containing compounds and redox-related quinones/anthraquinones. Further mechanistic studies determined that the redox state of the broad class of catechol-containing compounds is a key determinant of the amyloid inhibitor activities.

Conclusion: Our small molecule library screening platform was able to identify a broad class of amyloid inhibitors. Redox was found to be a key factor not only regulating the inhibitory activities but also involving the mechanism of inhibition. The molecular insights we gained not only explain why a large number of catechol-containing natural compounds, often enriched in healthy diet, have antineurodegeneration and anti-aging activities, but also could guide the rational design of therapeutic or nutraceutical strategies to target a broad range of neurodegenerative and related aging diseases.

\section{Background}

Protein aggregation diseases represent some of the most debilitating and increasingly more common aging-related human diseases. Alzheimer's, Parkinson's, prion diseases, and type 2 diabetes all fall under this category (Westermark et al, 2011; Knowles et al, 2014; Chiti \& Dobson, 2017; Wang et al, 2019; Wu et al, 2020). While there are currently no FDA approved anti-aggregation drugs available, data over the years continue to support the notion that one of the most attractive therapeutic targets against amyloidosis are the misfolding protein amyloids themselves (Eisenberg \& Jucker, 2012; Selkoe \& Hardy, 2016; Li \& Gotz, 2017). Insights gained from several latest high-resolution amyloid structures, and atomic details on their interactions with amyloid inhibitors have provided potentially exciting new paths towards the identification, characterization, and optimization of clinically viable inhibitor candidates (Fitzpatrick et al, 2017; Krotee et al, 2017; Li et al, 2017; Seidler et al, 2018; Spanopoulou et al, 2018; Griner et al, 2019;

Armiento et al, 2020a). Key to this development is achieving a better understanding of protein aggregation inhibition mechanisms and processes. Recent investigations have begun to elucidate some of the covalent and non-covalent mechanisms for small molecules or peptide-based inhibitors interacting 
with amyloidogenic proteins at various stages of protein aggregation formation (Velander et al, 2017; Seidler et al, 2018; Armiento et al, 2020b; Griner et al, 2019). Examples include stabilizing the soluble native confirmations of some amyloidogenic proteins (Johnson et al, 2012), perturbing or "remodeling" unaggregated or pre-aggregated amyloid species towards forming presumably innocuous, nonamyloidogenic aggregates (Ehrnhoefer et al, 2008; Hong et al, 2008; Bieschke et al, 2010), or even accelerating amyloid formation (Jha et al, 2016). However, in many cases, chemical classes of the inhibitors and a clear link between the chemical mode of action of an inhibitor and its macromolecular partner are poorly defined. Moreover, the biochemical, biophysical and pharmacological underpinnings of inhibitor-perturbed amyloid aggregation pathways or alternative aggregate assemblies are not always clear.

To systematically identify broad classes of small molecules and chemical types of protein aggregation inhibitors, we screened a US National Institutes of Health Clinical Collection (NIHCC) drug-repurposing library of 700 drugs and/or investigational compounds with diverse molecular scaffolds or chemical structures. We employed the thioflavin T (ThT) fluorescence-based assay to monitor toxic amyloid formation utilizing a 384-well plate semi high throughput screening platform (Cao et al, 2012; Brumshtein et al, 2015; Seidler et al, 2018; Fig. S1D). To make our findings broadly applicable, we screened in parallel against three prototype amyloidogenic proteins: amylin, $A \beta$ (Figure S1A) as well as 2 N4R tau (Spillantini \& Goedert, 2013). Amylin amyloidosis and plaque deposition in the pancreas are hallmark features of type 2 diabetes (Verchere et al, 1996; Westermark et al, 2011), whereas A $\beta$ plaques and tau neurofibrillary tangles are well-established neuropathogenic biomarkers for Alzheimer's disease.

\section{Methods}

\section{Peptides and Chemicals}

Synthetic amidated human amylin was purchased from AnaSpec Inc. (Fremont, CA) and the peptide quality was further validated by the Virginia Tech Mass Spectrometry Incubator. Hexafluroisopropanol (HFIP) and thioflavin T (ThT) were purchased from Sigma Aldrich (St. Louis, MO). Small molecule amyloid inhibitors and relevant control compounds were purchased from Toronto Research Chemicals (North York, ON, Canada), Fisher Scientific Inc. (Hampton, NH), Sigma-Aldrich Corp. (St. Louis, MO) or Cayman Chemical Company (Ann Arbor, MI). Dulbecco's phosphate buffer saline (DBPS) pH 7.4, was purchased from Lonza (Walkersville, MD). Black 96-well non-stick-clear-bottom plates and optically clear sealing film were purchased from Greiner Bio-one (Germany) and Hampton Research (Aliso Viejo, CA) respectively. 300 mesh formvar-carbon-coated copper grids and uranyl acetate replacement solution (UAR) were purchased from Electron Microscopy Sciences (Hatfeild, PA).

Lyophilized amylin powder ( $0.5 \mathrm{mg}$ ) was initially dissolved in 100\% HFIP at a final concentration of 1$2 \mathrm{mM}$. The additional lyophilizing step was employed to eliminate traces of organic solvents, which have been shown to affect amylin aggregation. Aliquots were either lyophilized again prior to use in cell-based assays or dissolved directly into DPBS, $10 \mathrm{mM}$ phosphate buffer $\mathrm{pH} 7.4$ or $20 \mathrm{mM} \mathrm{Tris-HCl} \mathrm{pH} 7.4$ for all 
amylin amyloid-related in vitro assays. All remaining 1-2 mM stocks in $100 \%$ DMSO were stored at $-80^{\circ} \mathrm{C}$ until later use. The lyophilized powder from all compounds and ThT were dissolved in DMSO (10 mM) and distilled water or relevant buffer $(1-4 \mathrm{mM})$. These stocks were stored at $-20{ }^{\circ} \mathrm{C}$ until later use. Residual DMSO in the final samples used for all in vitro assays ranged from $0-9.5 \%$. We determined that these DMSO concentrations had negligible effects on amylin amyloid aggregation as reflected by ThT fluorescence, TEM, PICUP assay, and inhibitor-induced amylin amyloid remodeling assays.

UV-Vis absorption spectra were collected on a Varian Cary 50 UV-Vis spectrophotometer. Equal concentration of catechol, norepinephrine, and rosmarinic acid was used for each compound. Aerobic and anaerobic conditions referred in each case as exposure to air or incubation in an anaerobic chamber (described below) at the specified duration.

\section{Thioflavin-T Fluorescence Screening Assays}

Fluorescence experiments were performed using a SpectraMax M5 plate reader (Molecular Devices, Sunnyvale, CA) as described in our previous works (Velander et al, 2016; Wu et al, 2017). All kinetic reads were taken in non-binding all black clear bottom Greiner plates covered with optically clear sheets to minimize the evaporation of the solution in each well and stirred for 10 seconds prior to each reading. All experiments were repeated three times using peptide stock solutions from the same lot.

\section{(a) Screening against amylin amyloid}

Amylin stock preparation was initiated by dissolving lyophilized human amylin powder in $100 \% \mathrm{HFIP}$. After at least 2 hours of incubation at room temperature, HFIP was removed and the resulting amylin powder was re-dissolved in $100 \%$ DMSO and quantified by a standard BCA micro plate assay (i.e. HFIP is extremely volatile and difficult to accurately pipette; thus, DMSO is a better solvent for peptide transfer. Initial HFIP dissolution was employed because it has been suggested to aid in removal of trace contaminants that may be present within lyophilized batches of peptide). Solutions of $400 \mu \mathrm{M}$ amylin (100\% DMSO) were subsequently diluted with ThT in 1X DPBS and aliquoted to reading plate wells containing $0.45 \mu \mathrm{L}$ of compound or vehicle buffer controls to a final volume of $15 \mu \mathrm{L}$. Final concentrations prior to amylin amyloid aggregation were $10 \mu \mathrm{M}$ amylin, $30 \mu \mathrm{M}$ compound and $30 \mu \mathrm{M}$ ThT. Aggregation reactions were incubated at $25^{\circ} \mathrm{C}$ until the approximated plateau fluorescent readings. All plates were continuously monitored for ThT fluorescence every 10 minutes (plates were shaken briefly prior to each read) at excitation $444 \mathrm{~nm}$ and emission $490 \mathrm{~nm}$ until the plateau phase was reached.

\section{(b) Screening against 2N4R tau}

Purified recombinant 2N4R tau (the largest and the most studied human tau isoform) initially dissolved in 100\% 1X PNE buffer, was buffer exchanged with 1X HEPES buffer (10 mM HEPES, $30 \mathrm{mM} \mathrm{NaCl}, \mathrm{pH}$ 7.4). Amyloid aggregation was initiated in the presence or absence of compound by diluting tau protein into the wells of the reading plates to a final $34.4 \mu \mathrm{M}$ of tau, $12.5 \mu \mathrm{M}$ ThT and $0.06 \mathrm{mg} / \mathrm{ml}$ of heparin (served to promote tau amyloid formation) in each well. For compound treated wells, final molar concentration of 
compound to tau was $2: 1$. Tau aggregation reactions were incubated at $37^{\circ} \mathrm{C}$ and the fluorescent readings were taken at excitation $444 \mathrm{~nm} /$ emission $490 \mathrm{~nm}$.

\section{(c) Screening against AB42 peptide}

In contrast to the ThT fluorescence assays for amylin and 2N4R tau, non-continuous, two-point measurements were taken to represent starting and ending (at plateau) fluorescent signals for $A \beta 42$. Unaggregated solutions of $A \beta 42$ were typically prepared by dissolving lyophilized $A \beta 42$ powder with $100 \%$ HFIP. Subsequent peptide quantification of this solution was estimated based on a standard micro plate BCA assay. Initial stock solution preparation of A 42 for the ThT assay were prepared by evaporating HFIP treated stocks followed by a modified two-step aqueous dissolution process as previously described: Herein, HFIP evaporated stocks of A 342 were dissolved in $60 \mathrm{mM} \mathrm{NaOH}$. Next, these solutions were further diluted in 1X DPBS such that the final solution consisted of approximately $2.4 \mathrm{mM}$ $\mathrm{NaOH}$ (i.e. 4\% $60 \mathrm{mM} \mathrm{NaOH}, 96 \%$ 1X DPBS). Immediately after the addition of 1X DPBS, $6.48 \mu \mathrm{L}$ aliquots of $A \beta 42$ and $0.52 \mu \mathrm{L}$ aliquots of compound or buffer treated controls were distributed to the ThT reading plates. All plates were sealed to prevent evaporation and allowed to incubate at $37^{\circ} \mathrm{C}$ until the estimated plateau of aggregation (24 hours). Next, the reading plates were centrifuged prior to receiving $14 \mu \mathrm{L}$ of a $45 \mu \mathrm{M}$ ThT solution prepared in $50 \mathrm{mM}$ Glycine- $\mathrm{NaOH}, 8.6 \mathrm{pH}$. Finally, all plates were read at excitation $444 \mathrm{~nm}$ /emission 490 in order to estimate plateau phase amyloid aggregation as indicated by ThT fluorescence. Note, the final concentration of A 42 prior to being titrated with ThT, was approximately 12 $\mu \mathrm{M}$, at a 3:1 molar ratio of compound to $A \beta 42$.

The NIH Clinical Collection (NIHCC) library contains a total of 700 FDA-approved drugs and investigational compounds with diverse chemical structures. These small molecules have a history of use in human clinical trials. The collection was assembled through the NIH Molecular Libraries and Imaging Initiative. The library was supplied in 96-well plates in DMSO. For screening, 384-well plate was used. Bravo liquid handler with 96-channel disposable tip head (Agilent Technologies, Wilmington, DE) was used to aid sample transfer. Total volume in each well was $15 \mu \mathrm{l}$. Residual DMSO (<5.4\%) in each well had negligible effect on the fluorescent signals.

\section{Aggregation Assays in Anaerobic Chamber}

All reagents including buffers, protein and compounds were made anaerobic by flushing with nitrogen prior to being placed within an anaerobic chamber (glove box). $\mathrm{H}_{2}$ gas mixed with inert gas is circulated through metal catalyst to remove $\mathrm{O}_{2}$ gas in the chamber. Oxygen level was maintained between 1550 ppm within the anaerobic chamber. Typical aggregating conditions within the chamber were maintained at room temperature $\left(25-30^{\circ} \mathrm{C}\right)$. All anaerobic chamber-based ThT fluorescence assays were discontinuous, two point measurements (starting point, ending or plateau point).

\section{ThT Fluorescence-Based Amyloid Remodeling Assays}

Similarly with regular ThT fluorescence amyloid formation assays, remodeling assays extended the monitoring of the fluorescence signals continuously after an inhibitor or a control compound was spiked 
into the aggregation system (amyloidogenic protein, ThT, buffer, heparin the case of tau protein). The volume of spiked compound was made to $5 \%$ of total sample volume in each well such that the baseline fluorescence signal was minimally changed.

\section{Transmission Electron Microscopy (TEM) Analysis}

TEM images were obtained by a JEOL 1400 microscope operating at $120 \mathrm{kV}$. Samples consisting of 30 $\mu \mathrm{M}$ amylin (20 mM Tris-HCl, 2\% DMSO, pH 7.4) in the presence of drug or vehicle control were incubated for $\geq 48$ hours at $37^{\circ} \mathrm{C}$ with agitation. Prior to imaging, $2-5 \mu \mathrm{L}$ of sample were blotted on a 200 mesh formvar-carbon coated grid for 5 minutes and then stained with uranyl acetate (1\%). Both sample and stain solutions were wicked dry (sample dried before addition of stain) by filter paper. Qualitative assessments of the amount of fibrils or oligomers observed were made by taking representative images following a careful survey of each grid (>15-20 locations on each grid were surveyed).

\section{Gel-Based Amyloid Remodeling Assay}

Vehicle control or specified compounds were spiked into freshly dissolved amylin samples (containing amylin and buffer). Thereafter amylin aggregation was allowed to proceed for 3 days. Final amylin concentration was $15 \mu \mathrm{M}$ that included $45 \mu \mathrm{M}$ of compound (drug:amylin molar ratio was 3:1). After 3 days, these samples were vacuum dried and re-dissolved in $6.5 \mathrm{M}$ urea containing $15 \mathrm{mM}$ Tris and $1 \mathrm{X}$ SDS Laemmeli sample buffer, boiled at $95^{\circ} \mathrm{C}$ for $5-10$ minutes and subjected to SDS-PAGE followed by Western blot analysis with anti-amylin primary antibody (T-4157, 1:5000, Peninsula Laboratories, San Carlos, CA). All gel-based amyloid remodeling assays were repeated at least twice.

\section{Photo-Induced Crosslinking of Unmodified Proteins (PICUP) Assay}

Amylin aliquots from a master mix in $10 \mathrm{mM}$ phosphate buffer, $\mathrm{pH} 7.4$ were added separately to $0.6 \mathrm{~mL}$ eppendorf tubes containing small molecule inhibitors or DMSO vehicle loaded controls. Crosslinking for each tube was subsequently initiated by adding tris(bipyridyl)Ru(II) complex (Ruby) and ammonium persulfate (APS) (Typical amylin:Rubpy:APS ratios were fixed at 1:2:20, respectively, at a final volume of 15-20 $\mu \mathrm{L}$ ), followed by exposure to visible light, emitted from a 150-Watt incandescent light bulb, from a distance of $5 \mathrm{~cm}$ and for a duration of 5 seconds. The reaction was quenched by addition of 1X SDS sample buffer. PICUP results were visualized by SDS-PAGE (16\% acrylamide gels containing $6 \mathrm{M}$ urea), followed by silver staining. Final concentrations for all PICUP reactions included $30 \mu \mathrm{M}$ of amylin and $150 \mu \mathrm{M}$ of each compound.

\section{Liquid Chromatography - Mass Spectrometry (LC-MS) Analysis}

Analyses were performed on a Waters Synapt G2-S HDMS interfaced with an Acquity I-Class UPLC system. Freshly dissolved (time 0 ) or aged (exposure to air for 72 or 96 hours) norepinephrine samples at a concentration of $37.5 \mu \mathrm{M}$ (diluted in a mixed solvent of 2:98, acetonitrile:water, $\mathrm{v} / \mathrm{v}$ ) were characterized 
by positive mode LC-MS for its oxidative products. Norepinephrine was reliably detected on the reverse phase column with a retention time of 0.7 minutes. Visual inspection of the chromatograms showed a new peak at retention time between 0.85-0.95 minutes in the 72 and 96 hour sample that was not present at 0 hours. Oxidized species (new peaks) were characterized by MS/MS analysis.

The binary solvent system was composed of $0.1 \%$ formic acid in water $(A)$ and $0.1 \%$ formic acid in acetonitrile (B). A 10-minute gradient was used for the analysis with the following conditions: initial and hold for $30 \mathrm{~s}$ at $1 \% \mathrm{~B}$, a linear gradient to $90 \% \mathrm{~B}$ at $8 \mathrm{~min}$, hold at $90 \% \mathrm{~B}$ to $8.5 \mathrm{~min}$ and return to initial condition at $9 \mathrm{~min}$. Sample volumes for MS analysis were between 2-4 $\mu$ and injected onto a Waters UPLC BEH C18 $(1.7 \mu \mathrm{m}, 2.1 \mathrm{~mm} \times 50 \mathrm{~mm})$ held at $35^{\circ} \mathrm{C}$ for MS analysis. Source conditions for the mass spectrometer were: capillary $2.8 \mathrm{kV}$, source temperature $125^{\circ} \mathrm{C}$, sample cone $30 \mathrm{~V}$, source offset $80 \mathrm{~V}$, desolvation gas $500 \mathrm{~L} / \mathrm{Hr}$, desolvation temperature $400^{\circ} \mathrm{C}$, cone gas $50 \mathrm{~L} / \mathrm{Hr}$, and nebulizer gas 6 par. The $\mathrm{m} / \mathrm{z}$ scan range was 50-1800 for MS analysis and 50-600 for MS/MS analysis and a collision energy ramp of $15-40 \mathrm{eV}$ was used for MS/MS analysis.

\section{Statistical Analysis}

All data are presented as the mean \pm S.E.M and the differences were analyzed with a one-way analysis of variance followed by Holm-Sidak's multiple comparisons (amylin kinetics) or unpaired Student's $t$ test. These tests were implemented within GraphPad Prism software (version 6.0). $p$ values $<0.05$ were considered significant.

\section{Results}

One of the salient findings from the screening is that catechols and redox-related quinones/anthraquinones represent a broad class of amyloid inhibitors. As shown in Fig. 1A, these molecules made up a substantial portion of the identified strong inhibitors, which were defined as exhibiting greater than three standard deviation units below the ThT RFU observed for buffer treated individual amyloidogenic protein controls (dotted line). Fully 13 out of 41 strong inhibitors (32\%) for amylin, 11 out of 22 (50\%) for tau (2N4R isoform), and 14 out of 29 (48\%) for $A \beta$ were catechols or quinone/anthraquinones (highlighted as red dots). In the screens against amylin amyloid, out of 22 catechols and quinones/anthraquinones from the NIHCC library, 21 of them exhibited significant amyloid inhibitory activities (Table S1) with isoproterenol being the exception, with only a weak inhibitory effect. The majority of these catechols and quinones/anthraquinones (16 out of 22 drugs) were further validated by an orthogonal biochemical assay, Photo-Induced Cross-linking of Unmodified Proteins (PICUP), which identified cross-linked oligomers (Figs. S1B and S3A), or by a biophysical method, transmission electron microscopy (TEM; Fig. 1C, Fig. S1C and Table S1).

Similar with amylin screening results, 17 out of 22 catechols and quinones/anthraquinones showed significant activities against tau 2N4R amyloid (Table S2), with a majority of them further validated by a ThT fluorescence-based tau amyloid remodeling assay, accomplished by spiking testing compounds into pre-formed tau amyloids (Fig. S3B). Fully 18 out of 22 catechols and quinones/anthraquinones exhibited 
significant inhibition against $A \beta$ amyloid, with nine of them validated previously by assays including TEM and atomic force microscopy (AFM) (Table S3). Chemical structures of catechols and quinones/anthraquinones of the top hits are shown (Fig. S2). Numerous hits such as idarubicin (Compound \#318), daunorubicin (Compound \#321), and rifapentine (Compound \#601), showed strong inhibitory effects to all three amyloidogenic proteins (boxed red dots in Fig. 1A), whereas a few hits displayed preferential inhibition, such as rutin (Compound \#548; Tables S1-S3), which preferentially inhibited amylin amyloid formation.

To test the hypothesis whether the catechol functional group alone inhibits amyloid formation, we tested catechol in secondary assays and compared it with its control analog phenol. Catechol demonstrated moderate, yet significant activities in amylin amyloid inhibition in both ThT fluorescence assays and TEM analyses, whereas phenol showed no such inhibitory effects in both assays (Figs. 1B and 1C). These data demonstrate that the catechol functional moiety possesses general anti-amyloid activities. Our finding is supported not only by individual catechol-containing inhibitor examples (Caruana et al, 2011; Sato et al, 2013; Velander et al, 2016; Wu et al, 2017), but also by a large-scale computational data mining study: In a fragment-based combinatorial library screening to identify molecular scaffolds to target certain amyloidogenic proteins in neurodegeneration (Joshi et al, 2016), the catechol group was identified as the fragment with the highest observed occurrence, present in nearly 4,500 compounds among the $16,850(27 \%)$ in the $A \beta$ small molecule library.

Another class of chemical structures enriched in our screens was the anthraquinones/quinones (redoxrelated to catechols) and tetracyclines. Anthraquinones were previously observed to inhibit tau aggregation (Pickhardt et al, 2005) and quinones were reported to inhibit insulin oligomerization as well as fibril formation (Gong et al, 2014). With respect to tetracycline, our screen revealed that several variants were active in amyloid inhibition. This class of compounds was reported to also inhibit $A \beta$ and $\beta_{2}$-microglobulin amyloid fibrils (Forioni et al, 2001; Giorgetti et al, 2010).

Based on the fact that catechol-containing compounds and multiple anthraquinone/quinone compounds (redox related to catechols) exhibited strong anti-amyloid activities, we hypothesized that catechol autoxidation may be part of the general mechanism that significantly enhances the anti-amyloid activities of the catechol-containing compounds. To test this hypothesis, we compared the anti-amyloid activities of a collection of oxidized (or aged - exposed to the air for 48 hours) with non-oxidized (nonaged or freshly prepared) catechol-containing compounds. In virtually all cases, aged samples exhibited significantly greater activities than their identically prepared non-aged counterparts (Fig. 2A). Oxidationinduced activity enhancement was not observed with phenol nor a structurally similar but non-catechol amyloid inhibitor, morin. These combined results strongly suggested a catechol-dependent enhancement specificity, which was recapitulated under stringently defined aerobic/anaerobic conditions using an anaerobic chamber. Aerobic, but not anaerobic conditions, significantly enhanced anti-amyloid activities of several catecholamines and other catechol-containing inhibitors (Fig. 2D). The kinetic profiles of ThT fluorescence-based amylin amyloid inhibition showed significantly stronger inhibition with aged RA versus non-aged RA, with an even more dramatic inhibition activity enhancement was observed with aged 
norepinephrine (Figs. 2B \& 2C). Consistently, enhanced inhibition by norepinephrine occurred only under aerobic conditions (Fig. 2E), with marked reduction in fibril formation (Fig. 2F).

Multiple small molecule amyloid inhibitors, many of which are catechol-containing polyphenols, perturb or "remodel" unaggregated and/or pre-aggregated amyloid species into denaturant-resistant aggregates that displayed broad-range molecular weights; characterized as "smear-type" distributions on SDS-PAGE gels (Ehrnhoefer et al, 2008; Hong et al, 2008; Bieschke et al, 2010; Palhano et al, 2013; Wu et al, 2017). Using RA and norepinephrine as two representative cases, treatment with a reducing reagent such as cysteine nearly eliminated their amyloid remodeling activities in a dose dependent manner (Figs. 3A \& 3B). Similar effect was observed with glutathione and cystamine as well, and importantly, with other catechol-containing compounds including epigallocatechin gallate (EGCG) and dopamine, but not negative controls phenol and a non-catechol amyloid inhibitor, curcumin (Fig. 3C).

The chemical changes that occur during autoxidation were investigated by both UV-Vis and liquid chromatography-mass spectrometry (LC-MS) approaches. UV-Vis time course spectra confirmed that chemical changes were only detectable under aerobic conditions, and occurred coincidently with their enhanced anti-amyloid activities (Fig. 4A; highlighted by green asterisks). Such chemical changes were reflected in broad UV absorption spectra changes, particularly in the region of $300-350 \mathrm{~nm}$ (Fig. 4A). In an effort to identify the oxidized chemical species that contribute to the enhanced amyloid inhibition, we performed LC-MS analyses of aged norepinephrine. New species/peaks with increasing ion intensities over time (elution was collected at 0-96 hours) were detected in LC at the elution time between 0.85 min - $1.0 \mathrm{~min}$ (Fig. 4B). High-resolution MS identified four main ions within the norepinephrine sample ( $\mathrm{m} / \mathrm{z}$ peaks at $162.0548,164.0715,182.0458$, and 301.1037 in Fig. 4B). While the known oxidized product of norepinephrine, noradrenochrome $\left(\mathrm{C}_{8} \mathrm{H}_{5} \mathrm{NO}_{3}, 164.0342,[\mathrm{M}+\mathrm{H}]^{+}\right)$was anticipated product (Jimenez et al, 1984; Manini et al, 2007), the mass difference of 0.0373 from the observed 164.0715 does not support its presence. Thus all species remain unidentified. These ions may result from the acidic conditions of the LC separation as well as in-source reactions that may occur during the ionization process. Hence the features observed by LC-MS are presently considered signatures of the oxidation process and not the inhibitory species present in solution at neutral buffered $\mathrm{pH}$. Nonetheless, these ions were present in the time course of autoxidation that correlate with norepinephrine anti-amyloid activities as exhibited by both ThT and TEM assays (Figs. 2E \& 2F). These data agree well with studies that showed catecholamine oxidation products were effective anti-amyloidogenic agents against a-synuclein ( $\mathrm{Li}$ et al, 2004) and tau (Soeda et al, 2015). Collectively, our data demonstrate that autoxidation is a general pathway enhancing the anti-amyloid activities of catechol-containing compounds (Fig. 4C). In supporting this covalent inhibition model, we demonstrated the covalent conjugate adduct by high resolution mass spectrometry in our specific investigation on baicalein (Velander et al, 2016). Redox state is therefore a key factor that modulates the activities of a large number of catechol-containing amyloid inhibitors.

\section{Discussion}


A variety of covalent mechanisms can readily explain the observed effects of autoxidation for catecholmediated remodeling and the corresponding strong stability of the observed remodeled aggregates. Free radical cycling occurring during catechol autoxidation could directly cycle through nearby interacting amyloid proteins that subsequently lead to protein-protein and/or protein-compound adducts (Meng et al, 2009). Alternatively, remodeled aggregates may also form through covalent interactions between electrophilic o-quinone oxidized byproducts of catechol parent compounds and amyloid protein side chain amines (Zhu et al, 2004; Li et al, 2004; Hong et al, 2008; Meng et al, 2009; Popovych et al, 2012; Sato et al, 2013; Velander et al, 2016). Regardless of the exact mechanism, these data are consistent with the hypothesis that the presence of oxygen facilitates autoxidation, which in turn enables catecholcontaining compounds to engage in amyloid remodeling activities that leads to what are presumably innocuous off-pathway, non-toxic aggregates (Ehrnhoefer et al, 2008; Hong et al, 2008; Bieschke et al, 2010; Wu et al, 2017). It remains to be determined whether amyloid remodeling is merely phenomenological in nature or if it represents a key mechanism essential for inhibitor-mediated antiamyloid activities. Built from previous work (Zhu et al, 2004; Li et al, 2004; Pickhardt et al, 2005; Hong et al, 2008; Meng et al, 2009; Velander et al, 2016; Joshi et al, 2016; Velander et al, 2017; Wu et al, 2017), our data suggest that (i) catechol-containing compounds as well as redox related anthraquinones represent a broad class of amyloid inhibitors capable of targeting amyloid from multiple sequence-unrelated amyloidogenic proteins, and (ii) the redox states of catechol-containing compounds are a key determinant of their anti-amyloid activities.

We and several other research groups have shown that catechol groups in the catechol-containing polyphenols and flavonoids play key roles in amyloid inhibition (Caruana et al, 2011; Sato et al, 2013; Soeda et al, 2015; Velander et al, 2016; Wu et al, 2017). Significantly beyond from individual compounds, we have shown here systematically that catechols and redox-related quinones/anthraquinones are a broad class of amyloid inhibitors. To our knowledge, identification of a class of compounds using systematic screens against multiple prototype amyloidogenic proteins has yet to be reported. The catechol moiety is a common structural component of many natural products, broadly classified as polyphenols, many of which have anti-amyloid and anti-aging activities (Cao \& Raleigh, 2012; Ono et al, 2012; Yamada et al, 2015; Velander et al, 2017). Our results thus provide mechanistic insight into why this class of large number of natural compounds, often enriched in healthy diet, is active in neutralizing toxic amyloids, suggesting a combination of redox and structural modeling is a viable path to new antiamyloid structures with enhanced activities. Catechol-containing natural products offer the advantage of low cost and potentially low side effects, inasmuch offering significant potential as antineurodegeneration, anti-aging nutraceuticals.

Catechol and related quinone motifs are classified by Baell et al. as Pan Assay INterference compoundS (or PAINS) in drug discovery (Baell \& Holloway, 2010; Baell, 2016; Baell \& Nissink, 2018). However, validity of such overly broad classification is debatable as the authors conceded that about $5 \%$ of FDA-approved drugs contain PAINS-recognized substructures (Baell \& Nissink, 2018). For an example, EGCG, a wellestablished amyloid inhibitor (Ehrnhoefer et al, 2008; Bieschke et al, 2010; Palhano et al, 2013) that has undergone numerous clinical trials, falls into the PAINS category, because it contains the catechol motif. 
Nevertheless, caution has been exercised in our work to validate with multiple orthogonal in vitro and cellbased assays. ThT fluorescence assays for example, one of the most commonly used assays for screening amyloid inhibitors, could lead to false positives with certain hydroxyflavones (Noor et al, 2012). Orthogonal secondary assays, such as TEM analysis, PICUP, and cell-based assays, allowed us to follow up on the hits generated from ThT fluorescence-based primary screenings, minimizing such false positives.

Multiple structural studies suggest that there are different conformers and/or molecular polymorphism in multiple amyloidogenic proteins including amylin, $A \beta$, tau, $a-s y n u c l e i n$, and TDP-43 (Wiltzius et al, 2009; Tycko, 2015; Qiang et al, 2017; Guenther et al, 2018; Seidler et al, 2018; Cao et al, 2019; Arakhamia et al, 2020). Moreover, many studies indicate that unique amyloid conformers may faithfully propagate morphologically and biochemically between cells and tissues, in a prion-like manner (Frost et al, 2009; Watts et al, 2014; Strang et al, 2018). These experimental observations suggest that the accumulation and assembly of various protein aggregates in many protein misfolding diseases is not totally driven by the amino acid sequences of aggregation-prone molecules; instead, they may be governed by the precise cellular and pathological environment of aggregation conditions. It will be interesting to identify different conformers, key environmental factors, protein modifications, and correlate them with their cytotoxicity. Such structure and function classification will greatly facilitate the identification of specific pharmacophores for structure-based inhibitor design in the future.

\section{Conclusions}

In summary, we applied a molecular screening platform and identified catechol-containing compounds as a broad class of protein aggregation inhibitors against three prototypes of amyloidogenic proteins. We further discovered that the redox state is a key determinant of this broad class of aggregation inhibitor activities as part of a covalent conjugation mechanism. These molecular mechanistic insights not only explain why a large number of catechol-containing natural compounds, often enriched in healthy diet, have anti-neurodegeneration and anti-aging activities, but also could guide the rational design of therapeutic or nutraceutical strategies to target a broad range of increasingly prevalent neurodegenerative and related proteinopathies aging diseases.

\section{Abbreviations}

3,4-DHBA: 3,4-dihydroxybenzoic acid; 7,8-DHF: 7,8-dihydroxy flavone; Ab: amyloid b-peptide; AFM: atomic force microscopy; CA: caffeic acid; EGCG: epigallocatechin gallate; LC-MS: liquid chromatography-mass spectrometry; NIHCC: the NIH Clinical Collection library; Nor: norepinephrine; PICUP: photo-induced crosslinking of unmodified proteins; RA: rosmarinic acid; RFU: relative fluorescence unit; SAA: salvanic acid A; TCEP: tris(2-carboxyethyl)phosphine; TEM: transmission electron microscopy; ThT: thioflavin T.

\section{Declarations}


Acknowledgments

We thank Ms. Kathy Lowe at Virginia-Maryland Regional College of Veterinary Medicine for her excellent technical assistance in collecting TEM data. We thank Shradha Ladd for assistance in 2N4R tau recombinant protein purification. We thank Virginia Tech Center for Drug Discovery and Dr. Pablo Sobrado for instrument and NIHCC library access. We thank Prof. Jianyong Li for constructive discussions.

\section{Authors' contributions}

PV, LW, SBH performed the experiments, acquired and analyzed the data. NJV assisted with NIHCC library screening experiment and robotics usage. RFH designed LC-MS experiments and analyzed the data. BM, SZ contributed key reagents or facility and provided intellectual inputs in experimental design/method. BX conceived, organized, designed the experiments, and analyzed the data. BX, PV, LW, RFH wrote the paper. All authors read and approved the final manuscript.

\section{Availability of data and materials}

All data used and analyzed for the study are available from the corresponding author on reasonable request.

\section{Ethics approval and consent to participate}

Not applicable.

\section{Consent for publication}

Not applicable.

\section{Competing interests}

The author(s) declare that they have no competing interests.

\section{Funding}

This work was supported in part by NIH grants R03AG061531 (BX) and R01AG058673 (SZ), Alzheimer's Association/Michael J. Fox Foundation grant BAND-19-614848 (BX), Alzheimer's Drug Discovery Foundation 20150601 (SZ), the Hatch Program of the National Institute of Food and Agriculture, USDA (BX and RFH), Commonwealth Health Research Board Grant 208-01-16 (BX and SZ), Diabetes Action Research and Education Foundation Grant (BX), and the Alzheimer's and Related Diseases Research Award Fund of the Commonwealth of Virginia (Awards No. 16-1, 18-2 and 18-4 to BX, LW, and SZ).

\section{References}


1. Arakhamia T, Lee CE, Carlomagno Y, Duong DM, Kundinger SR, Wang K, Williams D, DeTure M, Dickson DW, Cook CN, Seyfried NT, Petrucelli L, Fitzpatrick AWP. Posttranslational Modifications Mediate the Structural Diversity of Tauopathy Strains. Cell. 2020;180:633-44.

2. Armiento V, Hille K, Naltsas D, Lin JS, Barron AE, Kapurniotu A. The Human Host-Defense Peptide Cathelicidin LL-37 is a Nanomolar Inhibitor of Amyloid Self-Assembly of Islet Amyloid Polypeptide (IAPP). Angew Chem Int Ed Engl. 2020a;59: 12837-41.

3. Armiento V, Spanopoulou A, Kapurniotu A. Peptide-based molecular strategies to interfere with protein misfolding, aggregation, and cell degeneration. Angew Chem Int Ed Engl. 2020b; 59:3372-84.

4. Baell JB, Holloway GA. New substructure filters for removal of pan assay interference compounds (PAINS) from screening libraries and for their exclusion in bioassays. J Med Chem. 2010;53:2719-40.

5. Baell JB. Feeling Nature's PAINS: Natural Products, Natural Product Drugs, and Pan Assay Interference Compounds (PAINS). J Nat Prod. 2016;79:616-28.

6. Baell JB, Nissink JWM. Seven Year Itch: Pan-Assay Interference Compounds (PAINS) in 2017-Utility and Limitations. ACS Chem Biol. 2018;13:36-44.

7. Bieschke J, Russ J, Friedrich RP, Ehrnhoefer DE, Wobst H, Neugebauer K, Wanker EE. EGCG remodels mature a-synuclein and amyloid- $\beta$ fibrils and reduces cellular toxicity. Proc Natl Acad Sci USA. 2010;107:7710-5.

8. Brumshtein B, Esswein SR, Salwinski L, Phillips ML, Ly AT, Cascio D, Sawaya MR, Eisenberg DS. Inhibition by small-molecule ligands of formation of amyloid fibrils of an immunoglobulin light chain variable domain. Elife. 2015;4:e10935.

9. Cao P, Raleigh DP. Analysis of the inhibition and remodeling of islet amyloid polypeptide amyloid fibers by flavanols. Biochemistry. 2012;51:2670-83.

10. Cao Q, Boyer DR, Sawaya MR, Ge P, Eisenberg DS. Cryo-EM structures of four polymorphic TDP-43 amyloid cores. Nat Struct Mol Biol. 2019;26:619-27.

11. Caruana M, Högen T, Levin J, Hillmer A, Giese A, Vassallo N. Inhibition and disaggregation of asynuclein oligomers by natural polyphenolic compounds. FEBS Lett. 2011;585:1113-20.

12. Chiti $F$ and Dobson CM. Protein misfolding, functional amyloid, and human disease: a summary of progress over the last decade. Annu Rev Biochem. 2017;86:27-68.

13. Ehrnhoefer DE, Bieschke J, Boeddrich A, Herbst M, Masino L, Lurz R, Engemann S, Pastore A, Wanker EE. EGCG redirects amyloidogenic polypeptides into unstructured, off-pathway oligomers. Nat Struct Mol Biol. 2008;15:558-66.

14. Eisenberg D, Jucker M. The amyloid state of proteins in human diseases. Cell. 2012;148: 1188-203.

15. Eleuteri S, Di Giovanni S, Rockenstein E, Mante M, Adame A, Trejo M, Wrasidlo W, Wu F, Fraering PC, Masliah E, Lashuel HA. Novel therapeutic strategy for neurodegeneration by blocking A $\beta$ seeding mediated aggregation in models of Alzheimer's disease. Neurobiol Dis. 2015;74:144-57.

16. Fitzpatrick AWP, Falcon B, He S, Murzin AG, Murshudov G, Garringer HJ, Crowther RA, Ghetti B, Goedert M, Scheres SHW. Cryo-EM structures of tau filaments from Alzheimer's disease. Nature. 
2017; 547:185-90.

17. Forloni G, Colombo L, Girola L, Tagliavini F, Salmona M. Anti-amyloidogenic activity of tetracyclines: studies in vitro. FEBS Lett. 2001;487:404-7.

18. Frost B, Diamond Ml. Prion-like mechanisms in neurodegenerative diseases. Nat Rev Neurosci. 2010;11:155-9.

19. Giorgetti S, Raimondi S, Pagano K, Relini A, Bucciantini M, Corazza A, Fogolari F, Codutti L, Salmona M, Mangione P, Colombo L, De Luigi A, Porcari R, Gliozzi A, Stefani M, Esposito G, Bellotti V, Stoppini M. Effect of tetracyclines on the dynamics of formation and destructuration of beta2-microglobulin amyloid fibrils. J Biol Chem. 2011;286:2121-31.

20. Gong H, He Z, Peng A, Zhang X, Cheng B, Sun Y, Zheng L, Huang K. Effects of several quinones on insulin aggregation. Sci Rep. 2014;4:5648.

21. Griner SL, Seidler P, Bowler J, Murray KA, Yang TP, Sahay S, Sawaya MR, Cascio D, Rodriguez JA, Philipp S, Sosna J, Glabe CG, Gonen T, Eisenberg DS. Structure-based inhibitors of amyloid beta core suggest a common interface with tau. Elife. 2019;8:e46924.

22. Guenther EL, Ge P, Trinh H, Sawaya MR, Cascio D, Boyer DR, Gonen T, Zhou ZH, Eisenberg DS. Atomic-level evidence for packing and positional amyloid polymorphism by segment from TDP-43 RRM2. Nat Struct Mol Biol. 2018;25:311-9.

23. Hasanbasic S, Jahic A, Berbic S, Znidaric MT, Zerovnik E. Inhibition of Protein Aggregation by Several Antioxidants. Oxid Med Cell Longev. 2018;8613209.

24. Hong DP, Fink AL, Uversky VN. Structural characteristics of alpha-synuclein oligomers stabilized by the flavonoid baicalein. J Mol Biol. 2008;383:214-23.

25. Jimenez M, Garcia-Canovas F, Garcia-Carmona F, Lozano JA, Iborra JL. Kinetic study and intermediates identification of noradrenaline oxidation by tyrosinase. Biochem Pharmacol. 1984;33:3689-97.

26. Jha NN, Ghosh D, Das S, Anoop A, Jacob RS, Singh PK, Ayyagari N, Namboothiri IN, Maji SK. Effect of curcumin analogs on alpha-synuclein aggregation and cytotoxicity. Sci Rep. 2016;6:28511.

27. Johnson SM, Connelly S, Fearns C, Powers ET, Kelly JW. The transthyretin amyloidoses: from delineating the molecular mechanism of aggregation linked to pathology to a regulatory-agencyapproved drug. J Mol Biol. 2012;421:185-203.

28. Joshi, P.; Chia, S.; Habchi, J.; Knowles, T. P. J.; Dobson, C. M.; Vendruscolo, M., A Fragment-Based Method of Creating Small-Molecule Libraries to Target the Aggregation of Intrinsically Disordered Proteins. ACS Comb Sci, 2016;18:144-53.

29. Knowles TPJ, Vendruscolo M, Dobson CM. The amyloid state and its association with protein misfolding diseases. Nat Rev Mol Cell Biol. 2014;15:384-96.

30. Krotee P, Rodriguez JA, Sawaya MR, Cascio D, Reyes FE, Shi D, Hattne J, Nannenga BL, Oskarsson ME, Philipp S, Griner S, Jiang L, Glabe CG, Westermark GT, Gonen T, Eisenberg DS. Atomic structures of fibrillar segments of hIAPP suggest tightly mated b-sheets are important for cytotoxicity. Elife. 2017;6. doi: 10.7554/eLife.19273. 
31. Li B, Ge P, Murray KA, Sheth P, Zhang M, Nair G, Sawaya MR, Shin WS, Boyer DR, Ye S, Eisenberg DS, Zhou ZH, Jiang L. Atomic structures of fibrillar segments of hIAPP suggest tightly mated $\beta$-sheets are important for cytotoxicity. Elife. 2017;6. pii: e19273. doi: 10.7554/eLife.19273.

32. Li C, Gotz J. Tau-based therapies in neurodegeneration: opportunities and challenges. Nature Rev Drug Discov 2017;16:863-83.

33. Li, J.; Zhu, M.; Manning-Bog, A. B.; Di Monte, D. A.; Fink, A. L., Dopamine and L-dopa disaggregate amyloid fibrils: implications for Parkinson's and Alzheimer's disease. FASEB J, 2004;18:962-4.

34. Manini P, Panzella L, Napolitano A, d'Ischia M. Oxidation chemistry of norepinephrine: partitioning of the O-quinone between competing cyclization and chain breakdown pathways and their roles in melanin formation. Chem Res Toxicol. 2007;20:1549-55.

35. Meng X, Munishkina LA, Fink AL, Uversky VN. Molecular mechanisms underlying the flavonoidinduced inhibition of alpha-synuclein fibrillation. Biochemistry 2009;48:8206-24.

36. Necula M, Kayed R, Milton S, Glabe CG. Small molecule inhibitors of aggregation indicate that amyloid beta oligomerization and fibrillization pathways are independent and distinct. J Biol Chem. 2007;282:10311-24.

37. Noor H, Cao P, Raleigh DP. Morin hydrate inhibits amyloid formation by islet amyloid polypeptide and disaggregates amyloid fibers. Protein Sci. 2012;21:373-82.

38. Ono K, Li L, Takamura Y, Yoshiike Y, Zhu L, Han F, Mao X, Ikeda T, Takasaki J, Nishijo H, Takashima A, Teplow DB, Zagorski MG, Yamada M. Phenolic Compounds Prevent Amyloid $\beta$-Protein Oligomerization and Synaptic Dysfunction by Site-specific Binding. J Biol Chem. 2012;287:14631-43.

39. Palhano FL, Lee J, Grimster NP, Kelly JW. Toward the Molecular Mechanism(s) by Which EGCG Treatment Remodels Mature Amyloid Fibrils. J Am Chem Soc. 2013;135:7503-10.

40. Pickhardt M, Gazova Z, von Bergen M, Khlistunova I, Wang Y, Hascher A, Mandelkow E M, Biernat J, Mandelkow E. Anthraquinones inhibit tau aggregation and dissolve Alzheimer's paired helical filaments in vitro and in cells. J Biol Chem. 2005;280:3628-35.

41. Popovych N, Brender JR, Soong R, Vivekanandan S, Hartman K, Basrur V, Macdonald PM, Ramamoorthy A. Site specific interaction of the polyphenol EGCG with the SEVI amyloid precursor peptide PAP(248-286). J Phys Chem. B 2012;116:3650-8.

42. Qiang W, Yau WM, Lu JX, Collinge J, Tycko R. Structural variation in amyloid- $\beta$ fibrils from Alzheimer's disease clinical subtypes. Nature. 2017;541:217-21.

43. Sato M, Murakami K, Uno M, Nakagawa Y, Katayama S, Akagi K, Masuda Y, Takegoshi K, Irie K. Sitespecific inhibitory mechanism for amyloid beta42 aggregation by catechol-type flavonoids targeting the Lys residues. J Biol Chem. 2013;288:23212-24.

44. Saunders JC, Young LM, Mahood RA, Jackson MP, Revill CH, Foster RJ, Smith DA, Ashcroft AE, Brockwell DJ, Radford SE. An in vivo platform for identifying inhibitors of protein aggregation. Nat Chem Biol. 2016;12:94-101.

45. Seidler PM, Boyer DR, Rodriguez JA, Sawaya MR, Cascio D, Murray K, Gonen T, Eisenberg DS. Structure-based inhibitors of tau aggregation. Nat Chem. 2018;10:170-6. 
46. Selkoe DJ, Hardy J. The amyloid hypothesis of Alzheimer's disease at 25 years. EMBO Mol Med. 2016;8:595-608.

47. Soeda Y, Yoshikawa M, Almeida OFX, Sumioka A, Maeda S, Osada H, Kondoh Y, Saito A, Miyasaka T, Kimura T, Suzuki M, Koyama H, Yoshiike Y, Sugimoto H, Ihara Y, Takashima A. Toxic tau oligomer formation blocked by capping of cysteine residues with 1,2-dihydroxybenzene groups. Nat Commun. 2015;6:10216.

48. Spillantini MG, Goedert M. Tau pathology and neurodegeneration. Lancet Neurol. 2013;12:609-22.

49. Spanopoulou A, Heidrich L, Chen HR, Frost C, Hrle D, Malideli E, Hille K, Grammatikopoulos A, Bernhagen J, Zacharias M, Rammes G, Kapurniotu A. Designed Macrocyclic Peptides as Nanomolar Amyloid Inhibitors Based on Minimal Recognition Elements. Angew Chem Int Ed Engl. 2018;57:14503-08.

50. Strang KH, Croft CL, Sorrentino ZA, Chakrabarty P, Golde TE, Giasson BI. Distinct differences in prionlike seeding and aggregation between Tau protein variants provide mechanistic insights into tauopathies. J Biol Chem. 2018;293:2408-21.

51. Tomiyama T, Shoji A, Kataoka K, Suwa Y, Asano S, Kaneko H, Endo N. Inhibition of amyloid beta protein aggregation and neurotoxicity by rifampicin. Its possible function as a hydroxyl radical scavenger. J Biol Chem. 1996;271:6839-44.

52. Tycko R. Amyloid polymorphism: structural basis and neurobiological relevance. Neuron. 2015;86:632-45.

53. Umeda T, Ono K, Sakai A, Yamashita M, Mizuguchi M, Klein WL, Yamada M, Mori H, Tomiyama T. Rifampicin is a candidate preventive medicine against amyloid- $\beta$ and tau oligomers. Brain. 2016;139:1568-86.

54. Velander P, Wu L, Ray WK, Helm RF, Xu B. Amylin Amyloid Inhibition by Flavonoid Baicalein: Key Roles of Its Vicinal Dihydroxyl Groups of the Catechol Moiety. Biochemistry 2016;55:4255-8.

55. Velander P, Wu L, Henderson F, Zhang S, Bevan DR, Xu B. Natural Product-Based Amyloid Inhibitors. Biochem Pharmacol. 2017;139:40-55.

56. Verchere CB, D'Alessio DA, Palmiter RD, Weir GC, Bonner-Weir S, Baskin DG, Kahn SE. Islet amyloid formation associated with hyperglycemia in transgenic mice with pancreatic beta cell expression of human islet amyloid polypeptide. Proc Natl Acad Sci U S A. 1996;93:3492-6.

57. Wang Z, Manca M, Foutz A, Camacho MV, Raymond GJ, Race B, Orru CD, Yuan J, Shen P, Li B, Lang Y, Dang J, Adornato A, Williams K, Maurer NR, Gambetti P, Xu B, Surewicz W, Petersen RB, Dong X, Appleby BS, Caughey B, Cui L, Kong Q, Zou WQ. Early preclinical detection of prions in the skin of prion-infected animals. Nat Commun. 2019;10:247.

58. Watts JC, Condello C, Stöhr J, Oehler A, Lee J, DeArmond SJ, Lannfelt L, Ingelsson M, Giles K, Prusiner SB. Serial propagation of distinct strains of $A \beta$ prions from Alzheimer's disease patients. Proc Natl Acad Sci U S A. 2014;111:10323-8.

59. Westermark P, Andersson A, Westermark GT. Islet amyloid polypeptide, islet amyloid, and diabetes mellitus. Physiol Rev, 2011;91:795-826. 
60. Wiltzius JJ, Landau M, Nelson R, Sawaya MR, Apostol MI, Goldschmidt L, Soriaga AB, Cascio D, Rajashankar K, Eisenberg D. Molecular mechanisms for protein-encoded inheritance. Nat Struct Mol Biol. 2009;16:973-8.

61. Wobst HJ, Sharma A, Diamond MI, Wanker EE, Bieschke J. The green tea polyphenol (-)epigallocatechin gallate prevents the aggregation of tau protein into toxic oligomers at substoichiometric ratios. FEBS Lett. 2015;589:77-83.

62. Wu L, Velander P, Liu D, Xu B. Olive Component Oleuropein Promotes beta-Cell Insulin Secretion and Protects beta-Cells from Amylin Amyloid-Induced Cytotoxicity. Biochemistry 2017;56:5035-9.

63. Wu L, Wang Z, Ladd S, Dougharty DT, Madhavan SS, Marcus M, Henderson F, Ray WK, Tan C, Siedlak S, Li J, Helm RF, Zhu X, Bloom GS, Zou WQ, Xu B. Human tau isoform aggregation and selective detection of misfolded tau from post-mortem Alzheimer's disease brains. bioRxiv. 2020; doi:10.1101/2019.12.31.876946.

64. Yamada M, Ono K, Hamaguchi T, Noguchi-Shinohara M. Natural Phenolic Compounds as Therapeutic and Preventive Agents for Cerebral Amyloidosis. Adv Exp Med Biol. 2015;863: 79-94.

65. Yu XL, Li YN, Zhang H, Su YJ, Zhou WW, Zhang ZP, Wang SW, Xu PX, Wang YJ, Liu RT. Rutin_inhibits_amylin-induced neurocytotoxicity and oxidative stress. Food Funct. 2015; 6:3296-306.

66. Zhu M, Rajamani S, Kaylor J, Han S, Zhou F, Fink AL. The flavonoid baicalein inhibits fibrillation of alpha-synuclein and disaggregates existing fibrils. J Biol Chem. 2004;279: 26846-57.

\section{Figures}



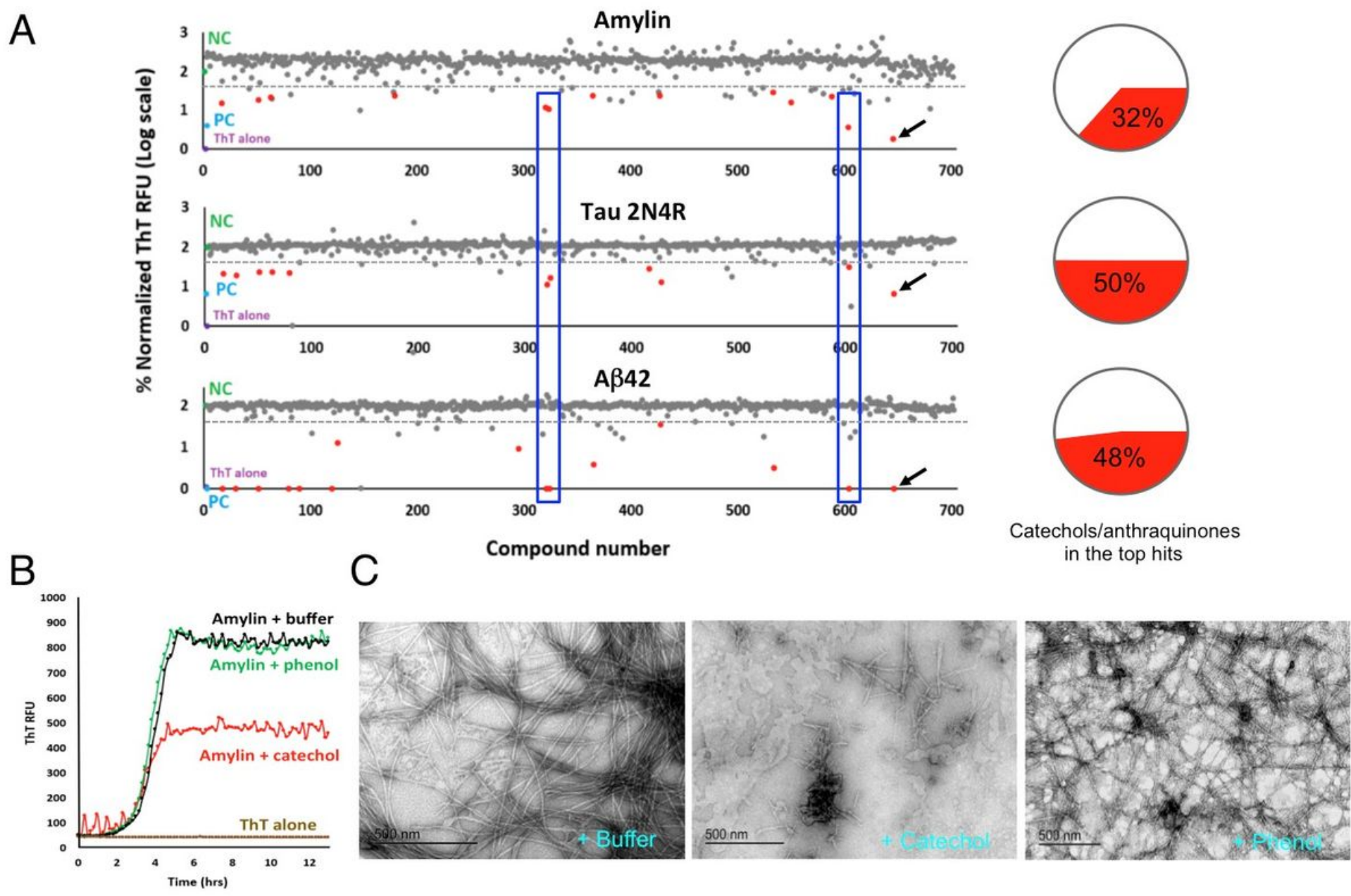

\section{Figure 1}

Diverse drug library screening identifies catechols and redox-related anthraquinones as a broad class of amyloid inhibitors. (A) Catechol-containing compounds or redox-related anthraquinones (shown as red dots) were observed to comprise $32 \%$ of the top hits (13 out of total 41 top hits) against amylin amyloid formation, $50 \%$ of the top hits (11 out of total 22 top hits) against 2 N4R isoform of human tau protein, and $48 \%$ of the top hits (14 out of total 29 top hits) against $A \beta 42$ amyloid. Top hits were defined by exhibiting a ThT fluorescent signal $\geq$ three standard deviations below buffer treated negative controls. Each dot represents a drug in the NIH Clinical Collection drug library (total 700 compounds). Buffer treated negative controls (NC), are shown in green. Positive controls (PC), amyloidogenic proteins treated with EGCG, are shown in blue. ThT alone, the assay background, is shown in cyan. Notice y-axis is shown in log-scale. A drug with a value of 2 in y-axis in the figure indicates that it has no inhibitory activity. Examples of common hits (drugs \#318, \#321, and \#601) for all three amyloid proteins are shown in blue boxes. One compound in the library was EGCG (red dots with black arrows), providing collaborating evidence of consistency of the screening method. (B) Catechol, but not phenol, demonstrates moderate yet significant anti-amylin amyloid effects as exhibited by ThT fluorescence assay. (C) TEM analyses validate that catechol, but not phenol, significantly reduces amylin fibril formation. Catechol's effect is 
moderate as reflected by the presence of some short fibrils. Typical molar ratios of compound to amyloidogenic protein were 2:1 for all ThT assays and 50:1 for the TEM assays.

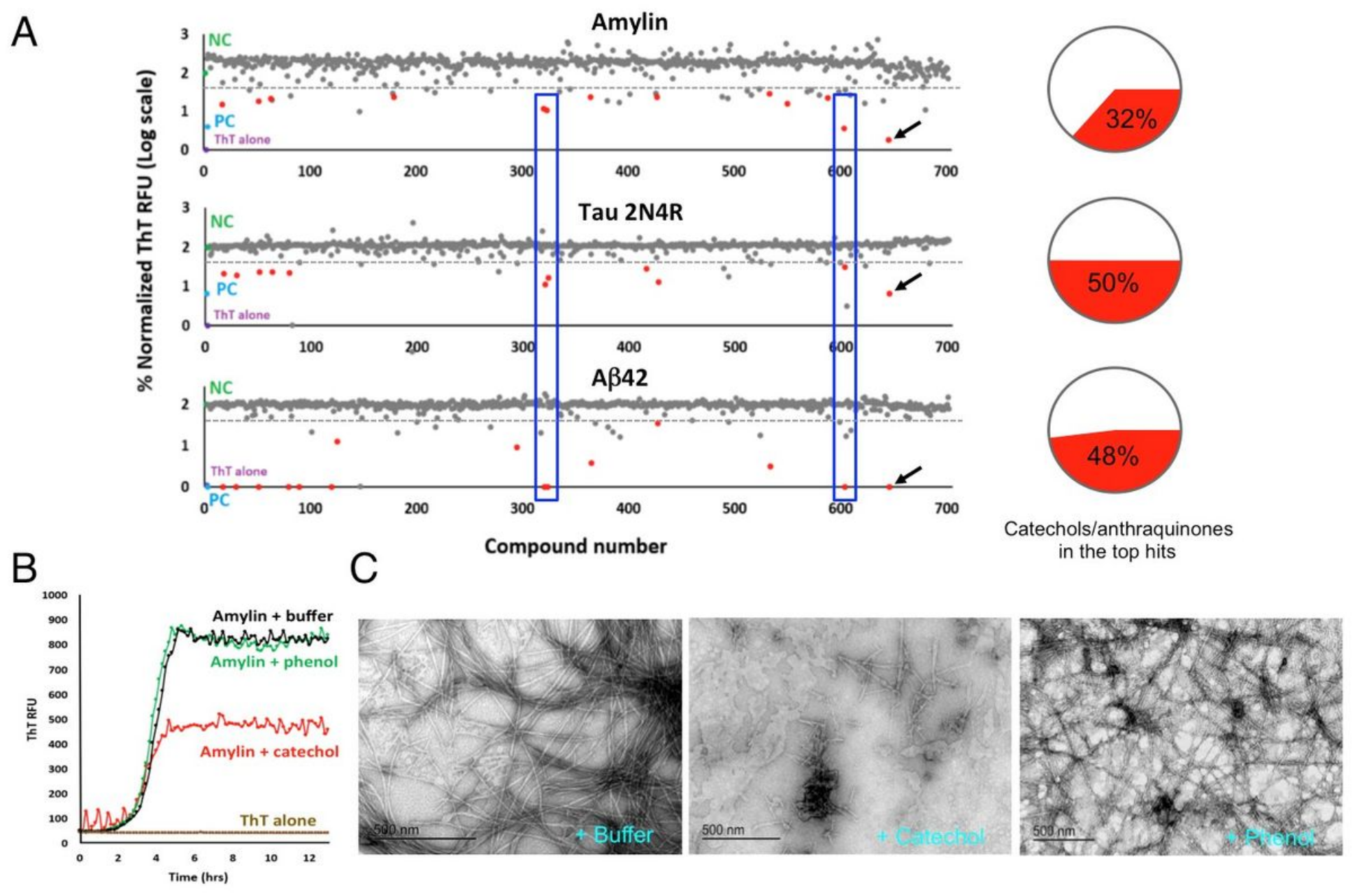

\section{Figure 1}

Diverse drug library screening identifies catechols and redox-related anthraquinones as a broad class of amyloid inhibitors. (A) Catechol-containing compounds or redox-related anthraquinones (shown as red dots) were observed to comprise $32 \%$ of the top hits (13 out of total 41 top hits) against amylin amyloid formation, $50 \%$ of the top hits (11 out of total 22 top hits) against 2 N4R isoform of human tau protein, and $48 \%$ of the top hits (14 out of total 29 top hits) against A 42 amyloid. Top hits were defined by exhibiting a ThT fluorescent signal $\geq$ three standard deviations below buffer treated negative controls. Each dot represents a drug in the $\mathrm{NIH}$ Clinical Collection drug library (total 700 compounds). Buffer treated negative controls (NC), are shown in green. Positive controls (PC), amyloidogenic proteins treated with EGCG, are shown in blue. ThT alone, the assay background, is shown in cyan. Notice y-axis is shown in log-scale. A drug with a value of 2 in $y$-axis in the figure indicates that it has no inhibitory activity. Examples of common hits (drugs \#318, \#321, and \#601) for all three amyloid proteins are shown in blue boxes. One compound in the library was EGCG (red dots with black arrows), providing collaborating evidence of consistency of the screening method. (B) Catechol, but not phenol, demonstrates moderate yet significant anti-amylin amyloid effects as exhibited by ThT fluorescence assay. (C) TEM analyses 
validate that catechol, but not phenol, significantly reduces amylin fibril formation. Catechol's effect is moderate as reflected by the presence of some short fibrils. Typical molar ratios of compound to amyloidogenic protein were 2:1 for all ThT assays and 50:1 for the TEM assays.
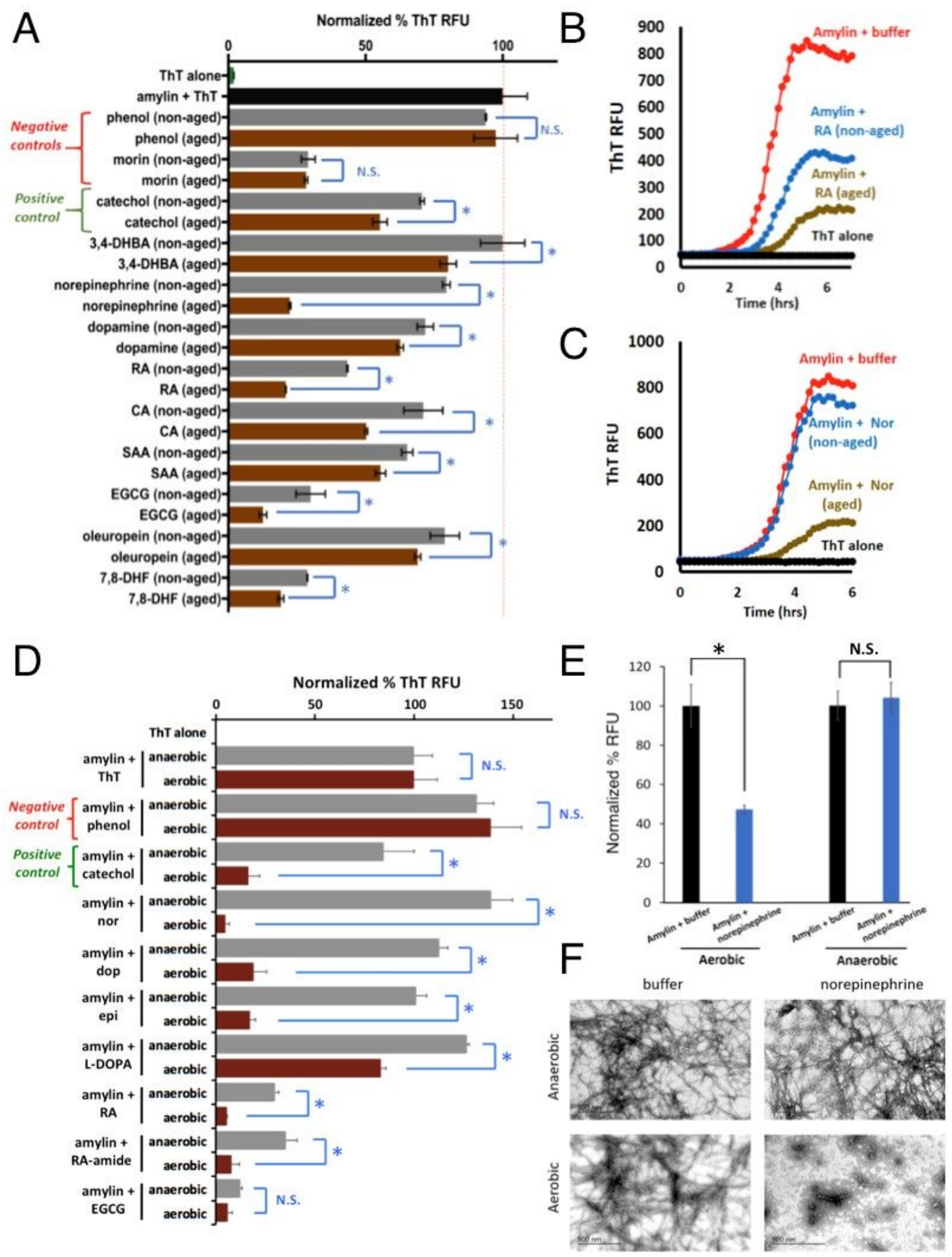

Figure 2

Autoxidation (or aerobic exposure), but not anaerobic conditions, significantly enhanced anti-amyloid activities associated with catechol-containing compounds. (A) Aged catechol-containing compounds (in 
brown), but not negative controls phenol (a non-inhibitor) or morin (an amylin amyloid inhibitor but with no catechol moiety) display enhanced anti-amyloid activities compared to their non-aged counterparts (in grey). All aged and non-aged treated amylin aggregation reactions were normalized to buffer treated amylin controls (black bar) indicated by the dashed red line. Abbreviations: 7,8-dihydroxy flavone (7,8DHF), rosmarinic acid (RA), caffeic acid (CA), salvanic acid A (SAA), 3,4-dihydroxybenzoic acid (3,4DHBA). Statistics were conducted using one-way ANOVA, multiple comparisons test; ${ }^{*}<0.05$. $(B, C)$ Representative ThT fluorescence assay time course of aged versus non-aged RA (panel $B$ ) and norepinephrine (panel C) treatments in amylin aggregations reactions shown in panel A. (D) Catecholcontaining compounds (in brown), showed significantly higher anti-amyloid activities under aerobic exposure compared to their anaerobic condition treated counterparts (in grey). All aerobic exposed and anaerobic condition treated amylin aggregation reactions were normalized to buffer treated amylin controls (amylin + ThT). Phenol served as a negative control and catechol as a positive control.

Abbreviations: norepinephrine: nor; dopamine: dop; epinephrine: epi; rosmarinic acid: RA; rosmarinic acid analog with an amide link, RA-amide; epigallocatechin gallate, EGCG. Statistics were conducted using one-way ANOVA, multiple comparisons test; ${ }^{*} \mathrm{p}<0.05$. (E,F) ThT and TEM assays confirm that the antiamylin amyloid activities of norepinephrine require aerobic exposure. Notice lack of fibril presence under aerobic condition with norepinephrine treatment. 

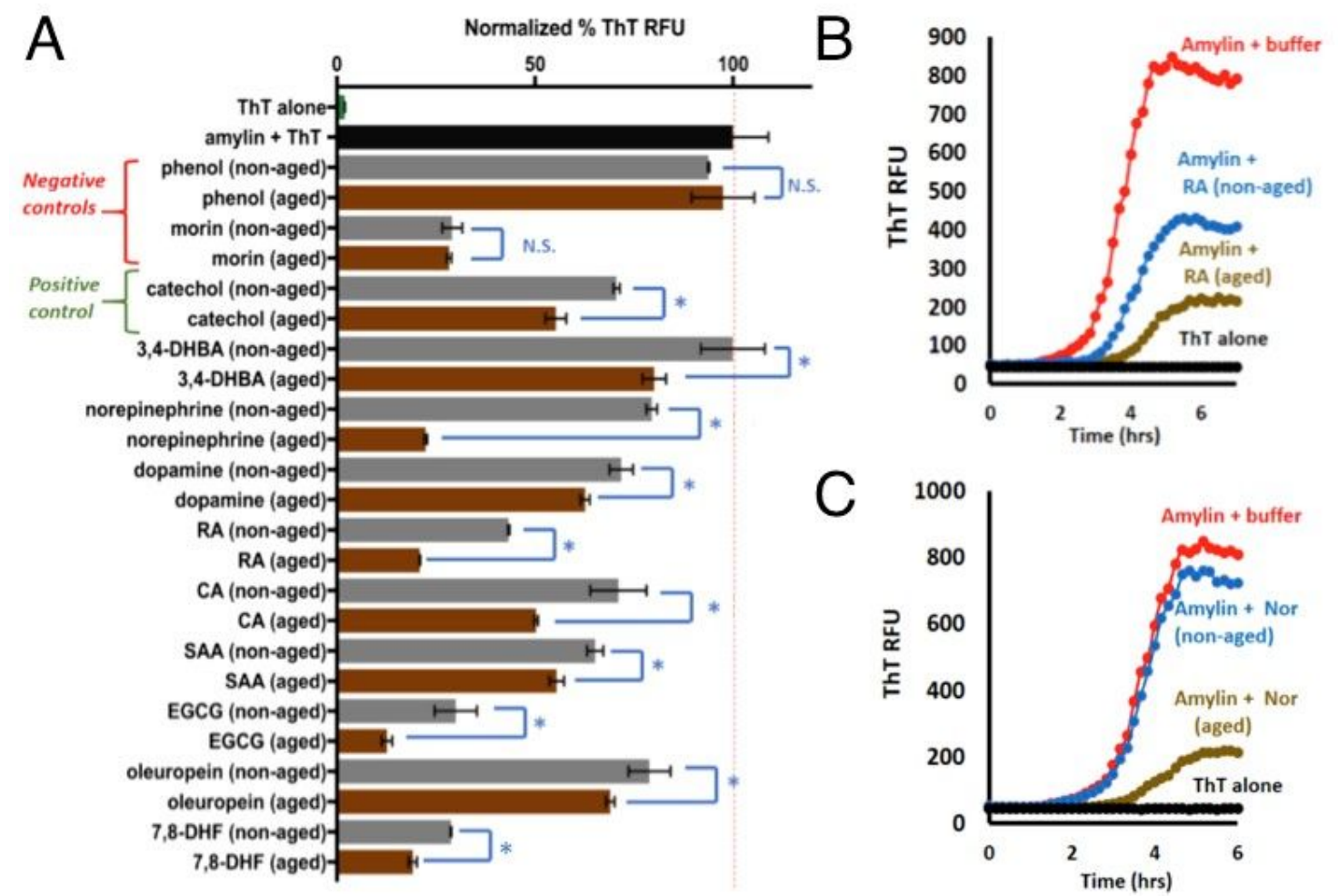

D

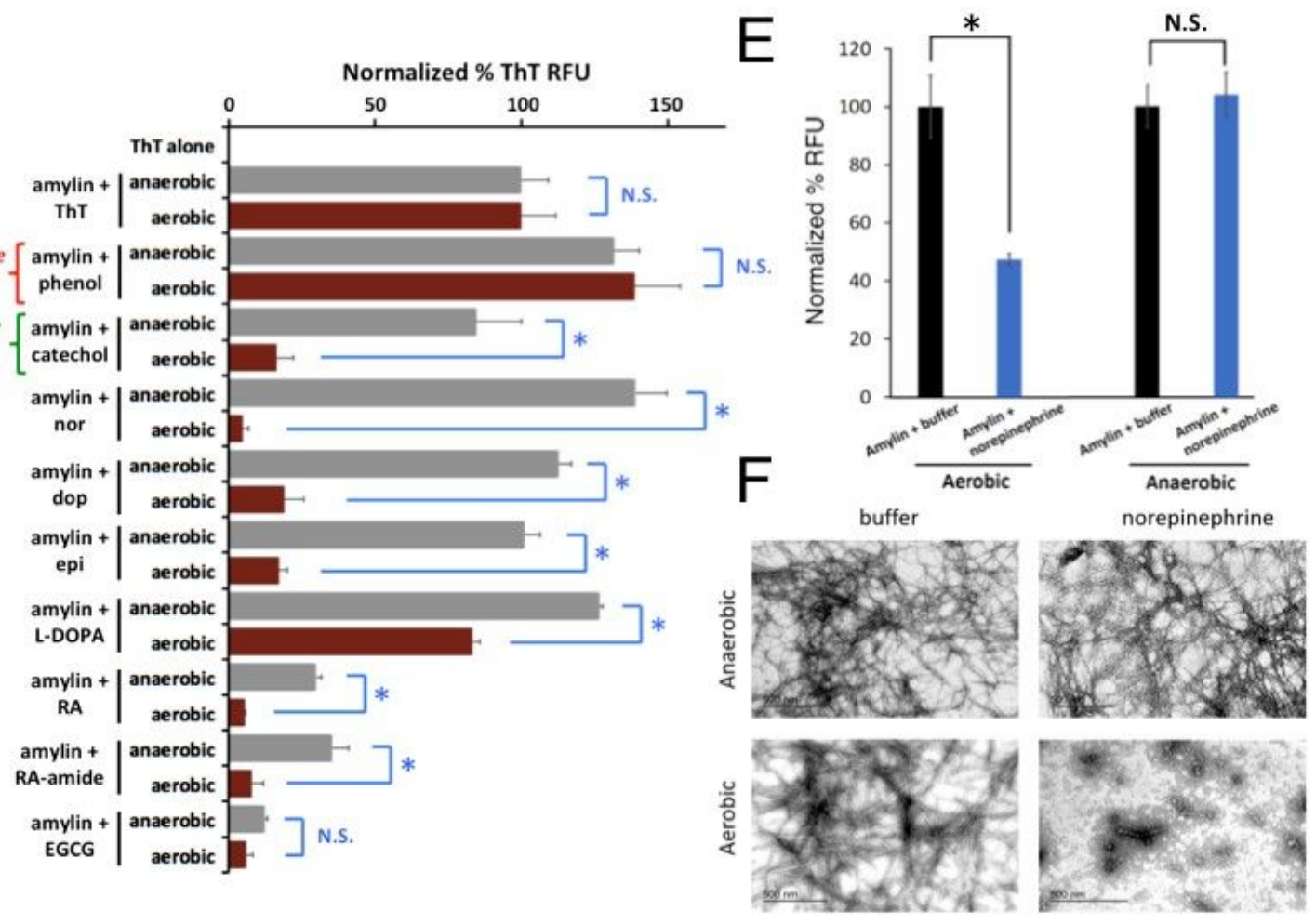

\section{Figure 2}

Autoxidation (or aerobic exposure), but not anaerobic conditions, significantly enhanced anti-amyloid activities associated with catechol-containing compounds. (A) Aged catechol-containing compounds (in brown), but not negative controls phenol (a non-inhibitor) or morin (an amylin amyloid inhibitor but with no catechol moiety) display enhanced anti-amyloid activities compared to their non-aged counterparts (in grey). All aged and non-aged treated amylin aggregation reactions were normalized to buffer treated 
amylin controls (black bar) indicated by the dashed red line. Abbreviations: 7,8-dihydroxy flavone (7,8DHF), rosmarinic acid (RA), caffeic acid (CA), salvanic acid A (SAA), 3,4-dihydroxybenzoic acid (3,4DHBA). Statistics were conducted using one-way ANOVA, multiple comparisons test; ${ }^{\star} p<0.05$. $(B, C)$ Representative ThT fluorescence assay time course of aged versus non-aged RA (panel $B$ ) and norepinephrine (panel C) treatments in amylin aggregations reactions shown in panel A. (D) Catecholcontaining compounds (in brown), showed significantly higher anti-amyloid activities under aerobic exposure compared to their anaerobic condition treated counterparts (in grey). All aerobic exposed and anaerobic condition treated amylin aggregation reactions were normalized to buffer treated amylin controls (amylin + ThT). Phenol served as a negative control and catechol as a positive control.

Abbreviations: norepinephrine: nor; dopamine: dop; epinephrine: epi; rosmarinic acid: RA; rosmarinic acid analog with an amide link, RA-amide; epigallocatechin gallate, EGCG. Statistics were conducted using one-way ANOVA, multiple comparisons test; ${ }^{\star} p<0.05$. (E,F) ThT and TEM assays confirm that the antiamylin amyloid activities of norepinephrine require aerobic exposure. Notice lack of fibril presence under aerobic condition with norepinephrine treatment. 
A
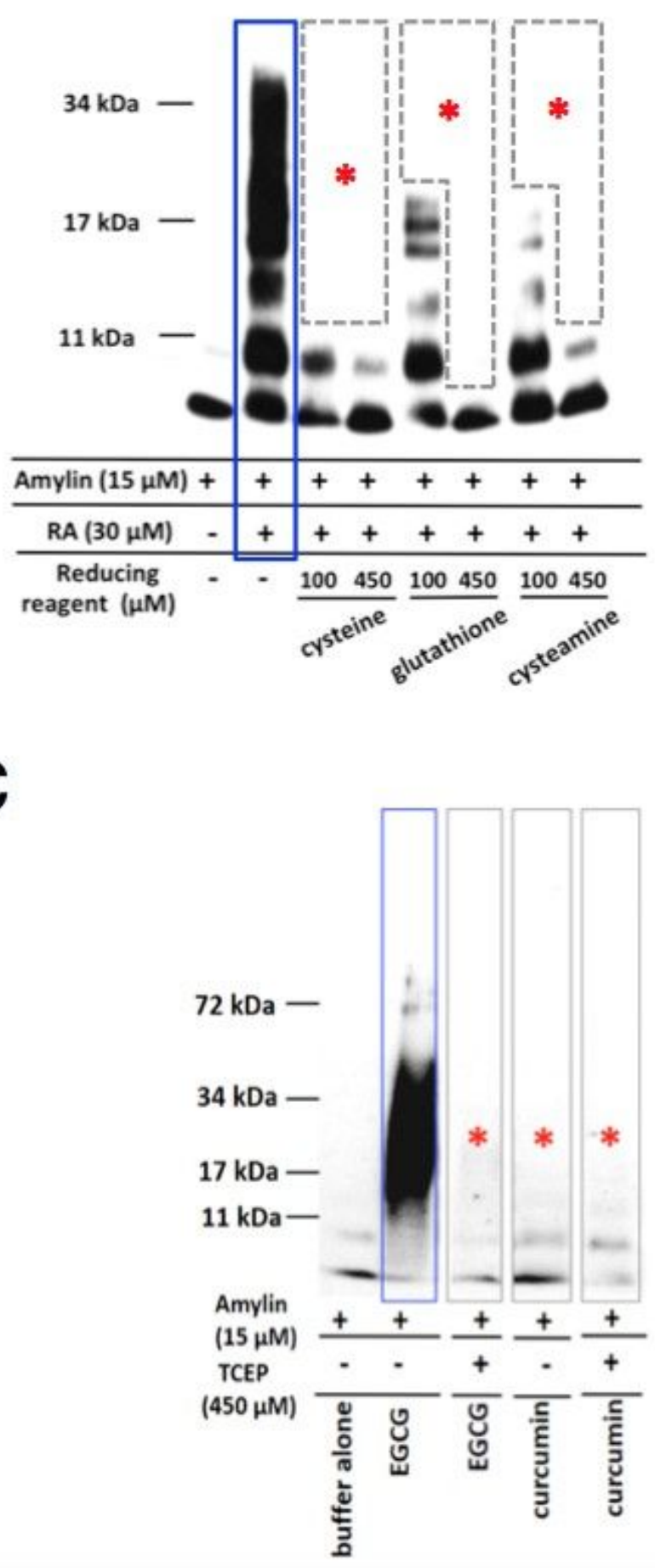

$\mathrm{B}$
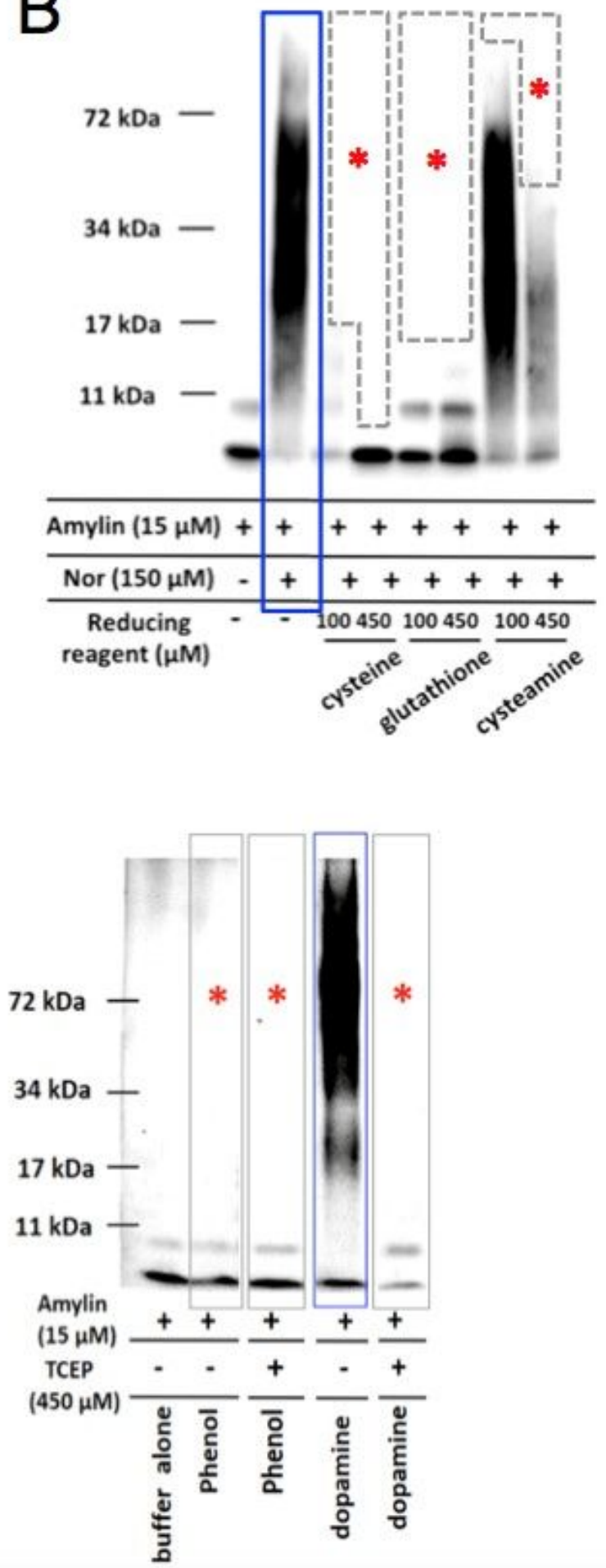

\section{Figure 3}

Blockage of amyloid remodeling activities of selective catechol-containing inhibitors by reducing agents. (A) Dose response effects of reducing agents cysteine, glutathione, and cysteamine on inhibiting RA (panel A) and norepinephrine (panel B) amyloid remodeling activities. Two different doses, $100 \mu \mathrm{M}$ and $450 \mu \mathrm{M}$ of reducing agents were used. (C) Control experiments demonstrate that reducing agent tris(2carboxyethyl)phosphine (TCEP) completely prevented the remodeling activities of amylin amyloid by 
catechol-containing inhibitors EGCG and dopamine but by neither a negative control, phenol, nor noncatechol inhibitor, curcumin. Amylin concentration used was $15 \mu \mathrm{M}$. The concentrations of reducing agent to inhibitors or control were 10:1 molar ratio (i.e., $450 \mu \mathrm{M}$ TCEP, $45 \mu \mathrm{M}$ of phenol, EGCG, or dopamine). In all panels, positive remodeling activities are highlighted in blue boxes and negative or mitigated remodeling activities are indicated by red asterisks.
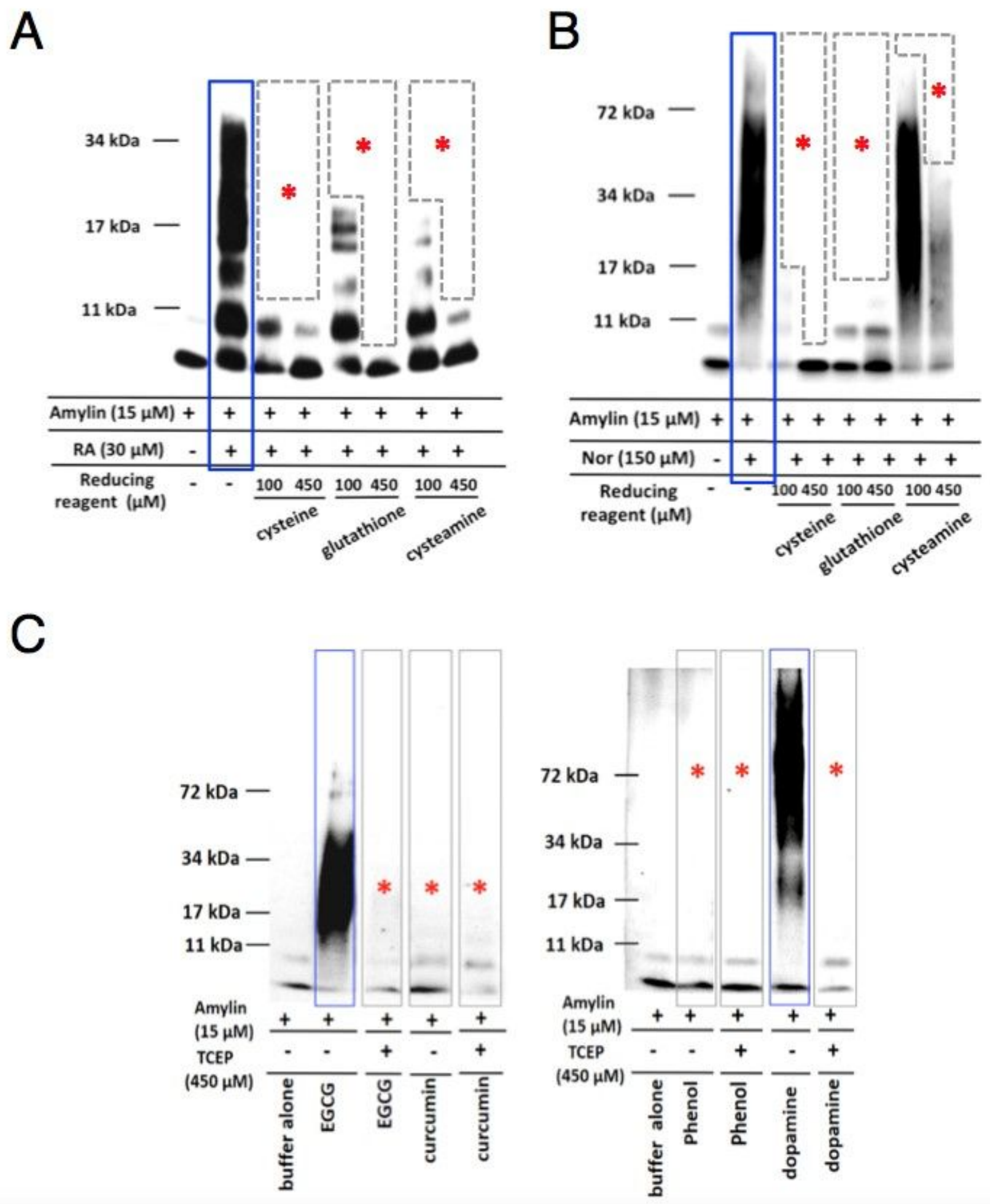

Figure 3 
Blockage of amyloid remodeling activities of selective catechol-containing inhibitors by reducing agents. (A) Dose response effects of reducing agents cysteine, glutathione, and cysteamine on inhibiting RA (panel A) and norepinephrine (panel B) amyloid remodeling activities. Two different doses, $100 \mu \mathrm{M}$ and $450 \mu \mathrm{M}$ of reducing agents were used. (C) Control experiments demonstrate that reducing agent tris(2carboxyethyl)phosphine (TCEP) completely prevented the remodeling activities of amylin amyloid by catechol-containing inhibitors EGCG and dopamine but by neither a negative control, phenol, nor noncatechol inhibitor, curcumin. Amylin concentration used was $15 \mu \mathrm{M}$. The concentrations of reducing agent to inhibitors or control were 10:1 molar ratio (i.e., $450 \mu \mathrm{M}$ TCEP, $45 \mu \mathrm{M}$ of phenol, EGCG, or dopamine). In all panels, positive remodeling activities are highlighted in blue boxes and negative or mitigated remodeling activities are indicated by red asterisks.
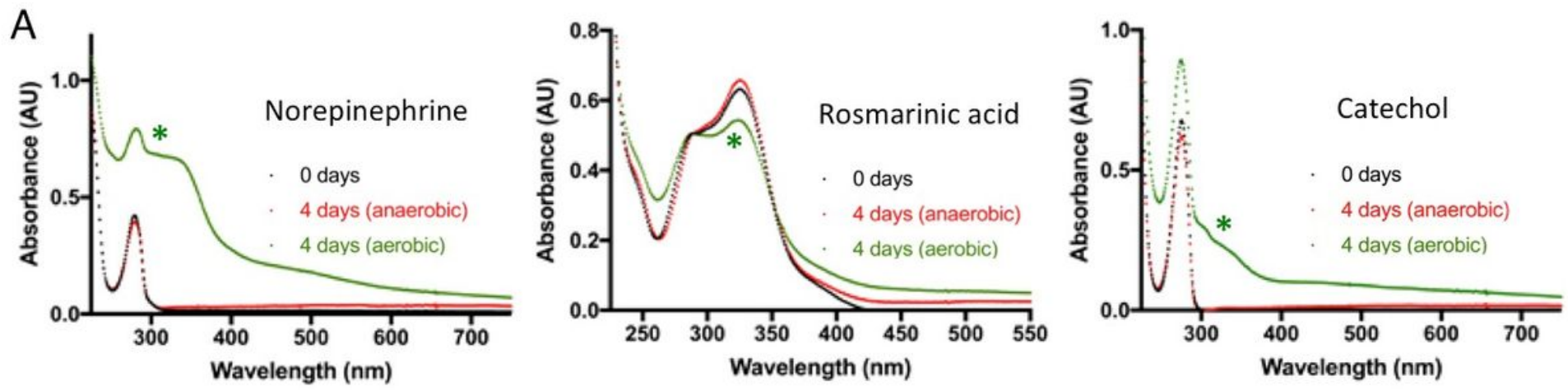

B
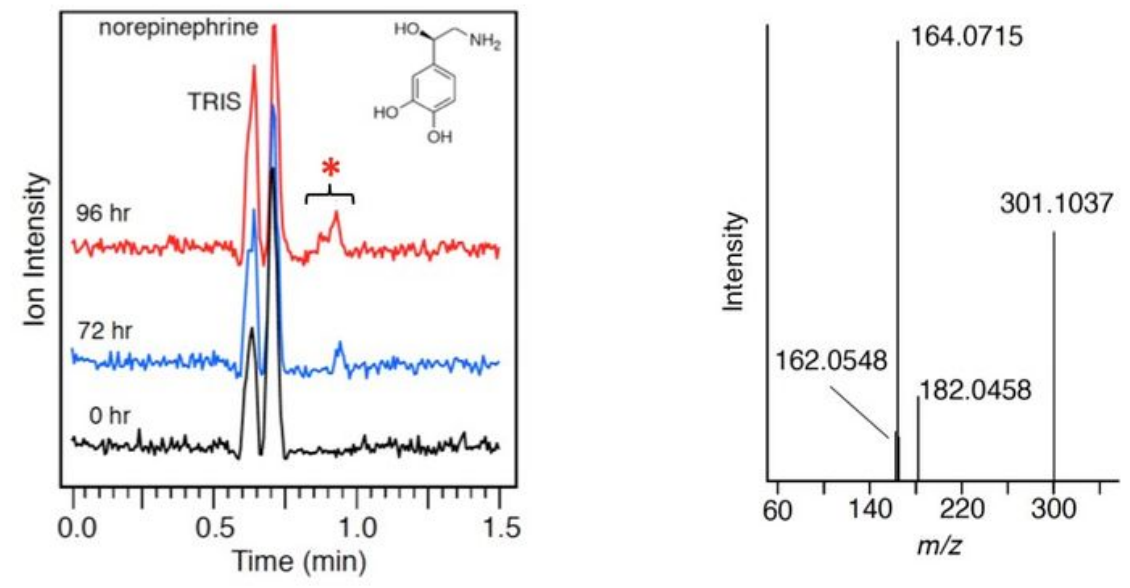

* norepinephrine autoxidized products

C

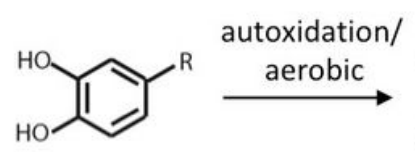

catechol

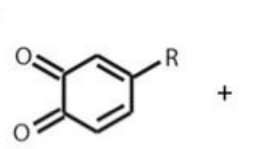

quinone-like intermediate amyloidogenic protein
Schiff base

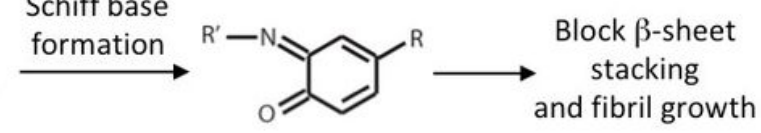

protein-inhibitor complex

\section{Figure 4}

UV-Vis and LC-MS characterization of oxidized products of selected catechols and model for amyloid inhibition by autoxidized catechol compounds. (A) Significant oxidative changes incurred for norepinephrine, rosmarinic acid, and catechol with 4 days exposure to aerobic condition, but not in anaerobic condition, in UV-Vis absorption spectra (indicated by green asterisks). (B) Freshly dissolved (time 0 ) or aged norepinephrine samples (72 or 96 hours) were characterized by LC-MS. Visual inspection 
of the chromatograms showed a new peak (highlighted by red asterisk) at $0.85-1.0$ minutes in the 72 and 96 hour sample that was not present at 0 hours. MS (positive mode) of this fraction identifies three species that are likely norepinephrine oxidized byproducts that include $[\mathrm{M}+\mathrm{H}+] 164.0715,182.0458$ and 182.0458. (C) A general model of how autoxidation or aerobic conditions enhance the activities of catechol-containing protein amyloid inhibitors, as we originally proposed in our specific investigation on baicalein (Velander et al, 2016).
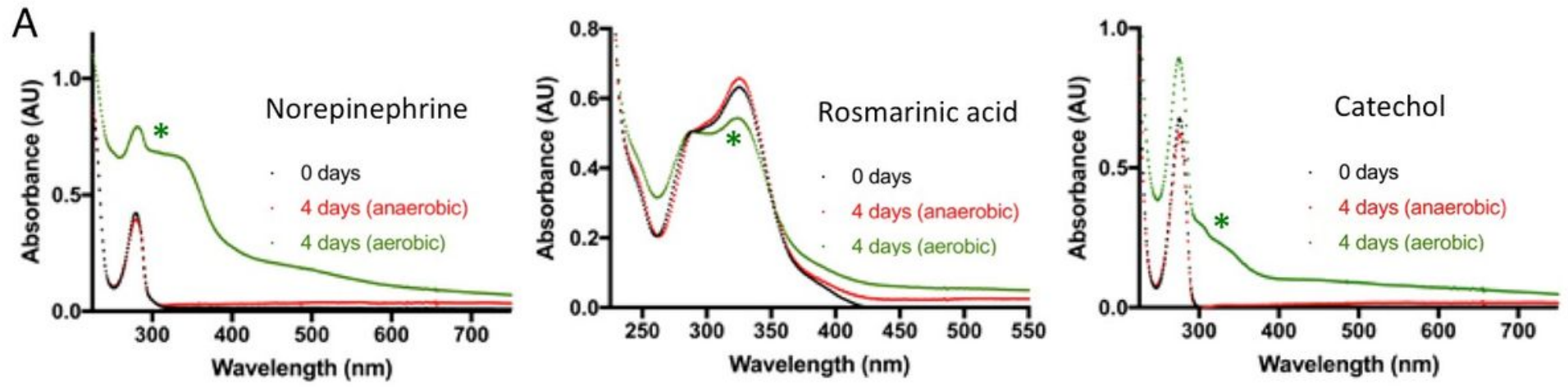

B
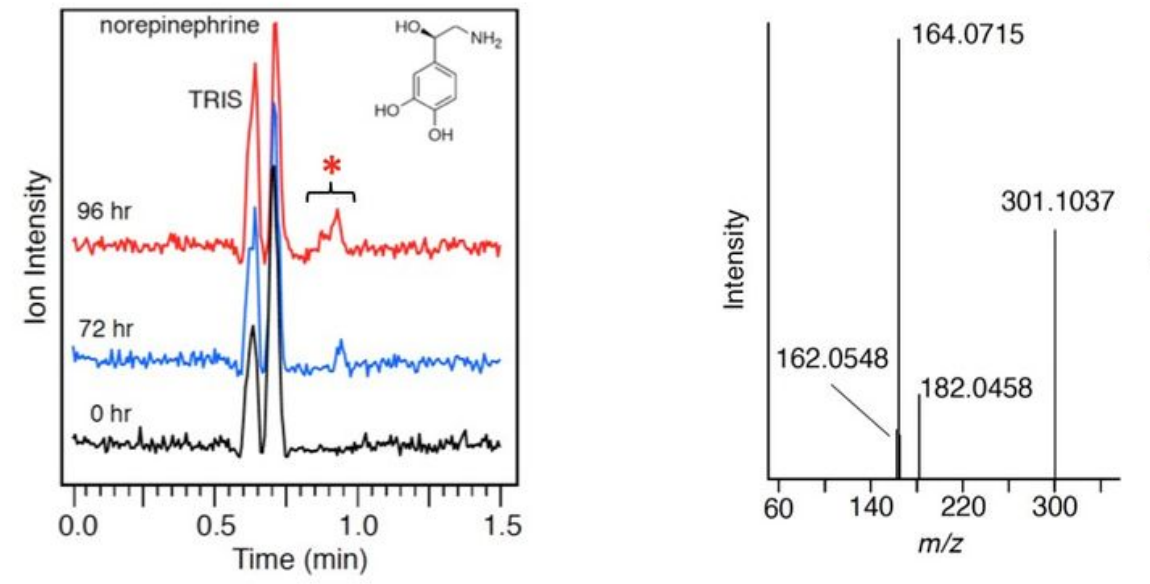

* norepinephrine autoxidized products

C

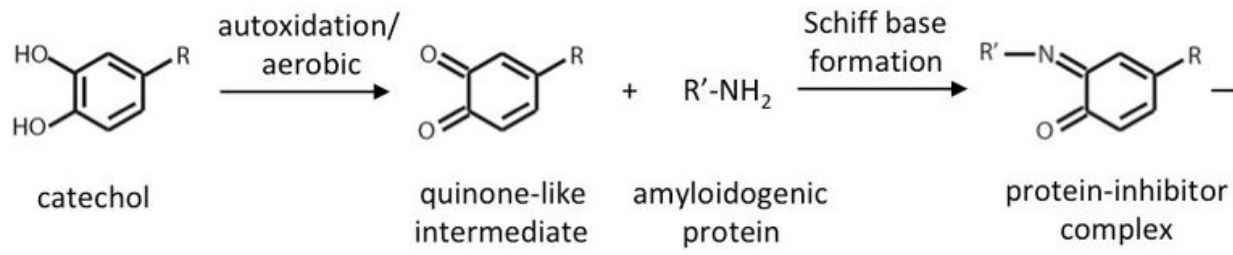

\section{Figure 4}

UV-Vis and LC-MS characterization of oxidized products of selected catechols and model for amyloid inhibition by autoxidized catechol compounds. (A) Significant oxidative changes incurred for norepinephrine, rosmarinic acid, and catechol with 4 days exposure to aerobic condition, but not in anaerobic condition, in UV-Vis absorption spectra (indicated by green asterisks). (B) Freshly dissolved (time 0 ) or aged norepinephrine samples (72 or 96 hours) were characterized by LC-MS. Visual inspection of the chromatograms showed a new peak (highlighted by red asterisk) at $0.85-1.0$ minutes in the 72 and 96 hour sample that was not present at 0 hours. MS (positive mode) of this fraction identifies three species that are likely norepinephrine oxidized byproducts that include $[\mathrm{M}+\mathrm{H}+] 164.0715,182.0458$ and 182.0458. (C) A general model of how autoxidation or aerobic conditions enhance the activities of 
catechol-containing protein amyloid inhibitors, as we originally proposed in our specific investigation on baicalein (Velander et al, 2016).

\section{Supplementary Files}

This is a list of supplementary files associated with this preprint. Click to download.

- SupplementalData.docx

- SupplementalData.docx 